\title{
Realimentação estática de saída de sistemas LPV positivos a tempo discreto *
}

\author{
Amanda Spagolla* Cecília F. Morais* \\ Ricardo C. L. F. Oliveira* Pedro L. D. Peres* \\ * Faculdade de Engenharia Elétrica e de Computação, \\ Universidade Estadual de Campinas - UNICAMP \\ Av. Albert Einstein, 400, 13083-852, Campinas, SP, Brasil \\ (e-mail: \{spagolla, cfmorais, ricfow, peres\}@dt.fee.unicamp.br).
}

\begin{abstract}
This paper proposes a new approach based on linear matrix inequalities (LMIs) to address the problem of static output-feedback control for positive linear parameter-varying (LPV) discrete-time systems. Unlike most of the LMI-based existing approaches for control of positive systems, that employ change of variables to obtain the gains, and impose a diagonal structure to the Lyapunov or the slack variable matrices to assure the closed-loop positivity of the system, the proposed technique is iterative and deals with the control gains directly as optimization variables of the problem. Therefore, structural constraints such as decentralization or bounds in the magnitude of the entries of the matrices that compose the control law are taken into account without introducing any extra conservativeness. The flexibility and advantages of the proposed approach are illustrated by means of numerical examples.

Resumo: Este artigo propõe uma nova abordagem baseada em desigualdades matriciais lineares (do inglês, Linear Matrix Inequalities - LMIs) para tratar o problema de controle por realimentação estática de saída de sistemas positivos lineares com parâmetros variantes discretos no tempo. Diferentemente da maioria das abordagens baseadas em LMIs para controle de sistemas positivos, que empregam mudanças de variáveis para obter os ganhos e recorrem ao uso de matrizes de Lyapunov ou variáveis de folga com estrutura diagonal para garantir a positividade do sistema em malha fechada, a técnica proposta é iterativa e trata os ganhos de controle diretamente como variáveis de otimização do problema. Dessa forma, restrições estruturais como descentralização ou limitações na magnitude dos elementos das matrizes que compõem a lei de controle são impostas sem introduzir nenhum conservadorismo adicional. A flexibilidade e as vantagens da abordagem proposta são ilustradas por meio de exemplos numéricos.
\end{abstract}

Keywords: Positive systems; Linear parameter-varying systems; Discrete-time systems; Linear matrix inequalities; Robust control.

Palavras-chaves: Sistemas positivos; Sistemas lineares variantes no tempo; Sistemas discretos no tempo; Desigualdades matriciais lineares; Controle robusto.

\section{INTRODUÇÃO}

Sistemas lineares com parâmetros variantes (do inglês, $L i$ near Parameter-Varying - LPV) representam uma classe particular de sistemas dinâmicos que têm despertado interesse crescente na literatura. Essa classe se caracteriza por uma modelagem versátil, capaz de representar sistemas lineares em que as matrizes do modelo de estado são afetadas por parâmetros variantes no tempo, ou sistemas variantes no tempo obtidos por meio de técnicas de identificação, ou ainda uma aproximação de sistemas nãolineares por um conjunto de modelos lineares (De Caigny et al., 2009; Kvieska et al., 2009; Hoffmann and Werner,

\footnotetext{
* O presente trabalho foi realizado com apoio da Coordenação de Aperfeiçoamento de Pessoal de Nível Superior - Brasil (CAPES) - Código de Financiamento 001, CNPq, FAPESP (Processos 2017/187855 e 2019/09363-5) e Projeto de P\&D PD-0063-3047/2018 - PA3047 da CPFL Energia.
}

2015). No que se refere à síntese de controladores para sistemas LPV, dois cenários principais se destacam: controle robusto (independente de parâmetros); e controle escalonado (dependente de parâmetros), que requer medições ou estimação dos parâmetros em tempo real e pode prover um melhor desempenho quando comparado ao controle robusto. Certamente a principal vantagem dos métodos da literatura que tratam parâmetros variantes no tempo em termos de modelos LPV politópicos ou afins (parâmetros pertencentes a hiper-retângulos) é a possibilidade de empregar métodos de otimização convexa descritos em termos de desigualdades matriciais lineares (do inglês, Linear Matrix Inequalities - LMIs) para testar a estabilidade robusta e projetar controladores ou filtros (Hoffmann and Werner, 2015). Uma característica particular dos métodos baseados em LMIs é que, em geral, as condições de projeto de um ganho robusto são um caso particular das condições de síntese de ganhos escalonados, bastando fixar algumas variáveis de otimização como independentes de 
parâmetros. As primeiras pesquisas que tratam análise e controle de sistemas LPV por meio de técnicas baseadas em LMIs empregam abordagens baseadas na estabilidade quadrática, com a matriz de Lyapunov constante (independente de parâmetros), gerando condições suficientes que, em geral, fornecem resultados conservadores. Apesar dos parâmetros variantes no tempo por vezes apresentarem taxas de variação limitadas, o cenário mais comumente investigado na literatura é quando os parâmetros variam arbitrariamente rápido. Nesse caso, o projeto de controle foi investigado empregando matrizes de Lyapunov com estruturas politópicas ou constantes por partes (Daafouz and Bernussou, 2001; Leite and Peres, 2004; Montagner et al., 2005) e, mais recentemente, utilizando condições LMIs descritas em termos de funções de Lyapunov dependentes do caminho (path dependent, em inglês) (Lee and Dullerud, 2006; Lee, 2006), generalizando os conceitos de estabilidade quadrática e matrizes de Lyapunov dependentes de parâmetros (Daafouz and Bernussou, 2001).

No contexto de sistemas lineares, existe uma ampla gama de problemas práticos que têm uma característica peculiar em comum: os modelos matemáticos possuem estados e saídas não-negativos para todas condições iniciais e entradas não-negativas (Dayawansa and Martin, 1999; Benzaouia and Tadeo, 2008). Tais sistemas, conhecidos na literatura como sistemas positivos (Farina and Rinaldi, 2000), podem ser encontrados nas áreas de engenharia (modelos de fluxo de redes), biologia e medicina (reprodução de bactérias e mutações virais), ciências sociais (modelos populacionais e armamentísticos), entre outras (Caccetta and Rumchev, 2000; Luenberger, 1979; Hernandez-Vargas et al., 2011). Por esse motivo, apesar dos métodos elaborados para sistemas lineares não poderem ser estendidos para essa classe de sistemas devido à restrição adicional de positividade, na última década surgiram diversos trabalhos na literatura que investigam os sistemas positivos nos contextos chaveado (Deaecto and Geromel, 2017b; Benzaouia and Tadeo, 2008; Hernandez-Vargas et al., 2011; Gurvits et al., 2007; Mason and Shorten, 2007; Shorten et al., 2009), cômputo de norma no domínio da frequência (Tanaka and Langbort, 2011; Najson, 2013), cálculo dos ganhos $L_{1}, L_{2}$, e $L_{\infty}$ (Rantzer, 2011; Ebihara et al., 2011, 2012; Briat, 2012), análise e controle $\mathcal{H}_{\infty}$ (Ebihara et al., 2014; Shen and Lam, 2017) ou $\mathcal{H}_{2}$ (Deaecto and Geromel, 2017a; Ebihara et al., 2018). No entanto, existem poucos trabalhos que tratam a estabilização de sistemas positivos LPV (Ait-Rami et al., 2014) e o controle por realimentação de saída (Bhattacharyya and Patra, 2018).

Este artigo propõe como contribuição um método de síntese de controladores estáticos por realimentação de saída (robustos ou dependentes de parâmetros) para sistemas LPV positivos em tempo discreto. Para isso, são propostas condições LMIs suficientes, resolvidas por meio de um procedimento iterativo baseado na técnica de Felipe et al. (2016), que tratam os ganhos de controle diretamente como variáveis de otimização (mudanças de variáveis não são necessárias). Assim, é possível garantir a positividade do sistema em malha fechada sem a necessidade de impor uma estrutura independente de parâmetros e diagonal à matriz de Lyapunov ou a qualquer outra matriz quadrada de recuperação do ganho. Essa estratégia facilita o projeto de controladores com estruturas particulares (descentra- lizados com elementos limitados em módulo ou norma, escalonados ou robustos) sem afetar as demais variáveis do problema (como a própria matriz de Lyapunov), as quais podem ser consideradas como dependentes de parâmetros. A existência de condições iniciais factíveis para o procedimento iterativo e uma estratégia de relaxação adotada a fim de reduzir o conservadorismo do método são discutidas. Além disso, soluções para os problemas de realimentação de estados e controle robusto, considerando o tratamento de sistemas lineares invariantes no tempo (do inglês, Linear Time-Invariant - LTI) incertos ou precisamente conhecidos, são obtidas por meio de adaptações imediatas do método proposto.

Notação: O espaço de matrizes reais com dimensão $n \times m$ é denotado por $\mathbb{R}^{n \times m}$. A transposta de uma matriz $M$ é representada por $M^{\prime}$, enquanto $\mathrm{He}(M)$ é usado para indicar $M+M^{\prime}$. Para matrizes simétricas $\left(M=M^{\prime}\right), M \succ$ $0(M \prec 0)$ denota que $M$ é definida positiva (negativa). Por outro lado, $M \geq 0$ indica que todos elementos da matriz $M$ são não-negativos. O símbolo * representa um bloco simétrico em uma matriz quadrada.

\section{PRELIMINARES}

Considere o sistema LPV positivo discreto no tempo

$$
\mathcal{G} \triangleq\left\{\begin{array}{l}
x(k+1)=A(\alpha(k)) x(k)+B(\alpha(k)) u(k), \\
y(k)=C_{y}(\alpha(k)) x(k)
\end{array}\right.
$$

em que $x(k) \in \mathbb{R}^{n_{x}}$ é o vetor de estados, $u(k) \in \mathbb{R}^{n_{u}}$ é a entrada de controle e $y(k) \in \mathbb{R}^{n_{y}}$ é a saída medida. As matrizes de espaço de estados de (1) são descritas como a combinação convexa de $N$ vértices conhecidos da forma

$$
M(\alpha(k))=\sum_{i=1}^{N} \alpha_{i}(k) M_{i}, \alpha(k) \in \Lambda,
$$

em que $\alpha(k)=\left[\alpha_{1}(k), \ldots, \alpha_{N}(k)\right]^{\prime}$ é um vetor de parâmetros variantes no tempo que pertence, para todo $k \geq 0$, ao simplex unitário descrito por

$$
\Lambda:=\left\{\zeta \in \mathbb{R}^{N}: \sum_{i=1}^{N} \zeta_{i}=1, \zeta_{i} \geq 0, i=1, \ldots, N\right\}
$$

e, por hipótese, $A(\alpha(k)) \geq 0, B(\alpha(k)) \geq 0$ e $C_{y}(\alpha(k)) \geq 0$ para todo $k \geq 0$. Este artigo aborda o problema de projeto de uma lei de controle por realimentação estática de saída dada por

$$
u(k)=L(\alpha(k)) y(k),
$$

para o sistema (1), de maneira que o sistema em malha fechada resultante, dado por

$$
\begin{aligned}
x(k+1) & =A_{c l}(\alpha(k)) x(k), \\
y(k) & =C_{y}(\alpha(k)) x(k)
\end{aligned}
$$

$\operatorname{com} A_{c l}(\alpha(k))=A(\alpha(k))+B(\alpha(k)) L(\alpha(k)) C_{y}(\alpha(k))$, seja positivo e assintoticamente estável. Antes de propor um método de projeto que resolva o problema de controle discutido neste artigo, é necessário apresentar algumas definições relacionadas a sistemas positivos discretos (Farina and Rinaldi, 2000).

Definição 1. O sistema (3) é positivo se possui estados e saídas não-negativos para toda entrada não-negativa e estado inicial não negativo.

Adicionalmente, a seguinte definição pode ser vista como uma extensão do conceito padrão de positividade no con- 
texto de sistemas LPV discretos descritos por equações de estado (Farina and Rinaldi, 2000).

Definição 2. O sistema em malha fechada (3) é positivo, se e somente se, as matrizes $A_{c l}(\alpha(k))$ e $C_{y}(\alpha(k))$ são nãonegativas para todo $\alpha(k) \in \Lambda, \forall k \geq 0$.

Finalmente, para que a lei de controle (2) possa garantir a estabilidade assintótica do sistema em malha fechada (3), uma condição LMI dependente de parâmetros para sistemas LPV discretos no tempo é apresentada a seguir (De Caigny et al., 2010).

Lema 1. O sistema (3) é assintoticamente estável se existir $P(\alpha(k))=P(\alpha(k))^{\prime} \succ 0$, tal que

$$
A_{c l}(\alpha(k)) P(\alpha(k)) A_{c l}(\alpha(k))^{\prime}-P(\alpha(k+1)) \prec 0,
$$

seja válida para todo $\alpha(k) \in \Lambda$ e $k \geq 0$.

\section{CONDIÇÃO DE SÍNTESE}

Uma das principais contribuições deste artigo é o próximo teorema, que apresenta uma condição LMI dependente de parâmetros suficiente para a síntese de controladores por realimentação estática de saída para sistemas LPV positivos discretos no tempo.

Teorema 1. Para dadas matrizes $Y_{i}(\bar{\alpha}(k)) \in \mathbb{R}^{n_{x} \times n_{x}}, i=$ $1,2,3$, se existirem matrizes dependentes de parâmetros $P(\alpha(k))=P(\alpha(k))^{\prime} \succ 0, X_{i}(\bar{\alpha}(k)) \in \mathbb{R}^{n_{x} \times n_{x}}, i=1,2,3$, e $L(\alpha(k)) \in \mathbb{R}^{n_{u} \times n_{y}}$ tais que

$$
\begin{gathered}
\mathcal{Q}(\bar{\alpha}(k))+\mathcal{X}(\bar{\alpha}(k)) \mathcal{B}(\bar{\alpha}(k))+\mathcal{B}^{\prime}(\bar{\alpha}(k)) \mathcal{X}^{\prime}(\bar{\alpha}(k)) \prec 0 \\
A_{c l}(\alpha(k)) \geq 0
\end{gathered}
$$

sejam válidas para todo $\bar{\alpha}(k)=(\alpha(k), \alpha(k+1)) \in \Lambda \times \Lambda$, em que

$$
\begin{gathered}
\mathcal{B}(\bar{\alpha}(k))=\left[\begin{array}{lll}
Y_{1}(\bar{\alpha}(k)) & Y_{2}(\bar{\alpha}(k)) & Y_{3}(\bar{\alpha}(k))
\end{array}\right], \\
\mathcal{X}(\bar{\alpha}(k))=\left[\begin{array}{lll}
X_{1}^{\prime}(\bar{\alpha}(k)) & X_{2}^{\prime}(\bar{\alpha}(k)) & X_{3}^{\prime}(\bar{\alpha}(k))
\end{array}\right]^{\prime}, \\
\mathcal{Q}(\bar{\alpha}(k))=\left[\begin{array}{ccc}
-P(\alpha(k+1)) & \star & \star \\
0 & P(\alpha(k)) & \star \\
A_{c l}^{\prime}(\alpha(k)) & -I & 0
\end{array}\right],
\end{gathered}
$$

$\operatorname{com} A_{c l}(\alpha(k))=A(\alpha(k))+B(\alpha(k)) L(\alpha(k)) C_{y}(\alpha(k))$, então $L(\alpha(k))$ é um ganho de controle escalonado por realimentação estática de saída que assegura a estabilidade assintótica e a positividade do sistema em malha fechada (3).

Prova: Note que, como $C_{y}(\alpha(k)) \geq 0$, segundo a Definição 2, a condição (6) assegura a positividade do sistema em malha fechada (3). Adicionalmente, se (5) é factível, $Y_{3}(\bar{\alpha}(k))$ tem posto completo e portanto $\mathcal{B}(\bar{\alpha}(k))$ pode ser reescrita como $\mathcal{B}(\bar{\alpha}(k))=Y_{3}(\bar{\alpha}(k))[-F(\bar{\alpha}(k))-G(\bar{\alpha}(k)) I]$ Em seguida, pré- e pós-multiplicando (5) respectivamente por $\mathcal{B}^{\perp \prime}(\alpha(k))$ e $\mathcal{B}^{\perp}(\alpha(k))$ com

obtém-se

$$
\mathcal{B}^{\perp^{\prime}}(\bar{\alpha}(k))=\left[\begin{array}{lll}
I & 0 & F^{\prime}(\bar{\alpha}(k)) \\
0 & I & G^{\prime}(\bar{\alpha}(k))
\end{array}\right]
$$

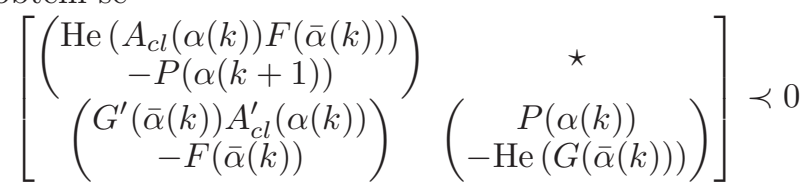

Multiplicando-se $(8)$ à direita por $N^{\prime}(\alpha(k))$ e à esquerda por $N(\alpha(k))=\left[I A_{c l}(\alpha(k))\right]$, tem-se $(4)$, que assegura a estabilidade assintótica do sistema (3).
O Teorema 1 pode ser adaptado para prover controladores escalonados por realimentação de estados, como mostrado no seguinte corolário.

Corolário 1. Para dadas matrizes $Y_{i}(\bar{\alpha}(k)) \in \mathbb{R}^{n_{x} \times n_{x}}, i=$ $1,2,3$, se existirem matrizes dependentes de parâmetros $P(\alpha(k))=P(\alpha(k))^{\prime} \succ 0, X_{i}(\bar{\alpha}(k)) \in \mathbb{R}^{n_{x} \times n_{x}}, i=1,2,3$, e $L(\alpha(k)) \in \mathbb{R}^{n_{u} \times n_{x}}$ tais que (5) e (6) sejam válidas para todo $\bar{\alpha}(k)=(\alpha(k), \alpha(k+1)) \in \Lambda \times \Lambda$, em que $A_{c l}(\alpha(k))=A(\alpha(k))+B(\alpha(k)) L(\alpha(k))$, então $L(\alpha(k))$ é um ganho de controle escalonado por realimentação de estados que assegura a estabilidade assintótica e a positividade do sistema (1) em malha fechada.

O Teorema 1 e o Corolário 1 podem ser adaptados para a síntese de controladores robustos para sistemas LTI incertos, como mostrado na seguinte observação. Note ainda que os resultados também aplicam-se a problemas de controle de sistemas discretos incertos desconsiderando a restrição de positividade (6).

Observação 1. Se o sistema (1) é LTI (matrizes $M(\alpha(k)=$ $M(\alpha), \forall k \geq 0, \alpha \in \Lambda$ ), então um controlador robusto (independente de parâmetros) $L$ que estabiliza o sistema (1) e assegura a positividade em malha fechada, é obtido resolvendo-se (5) e (6), com $\alpha(k+1)=\alpha(k)=\alpha$ (i.e., omitindo-se a dependência de $k$ das matrizes dependentes de parâmetros).

\section{PROCEDIMENTO ITERATIVO}

A condição (5) é linear nas variáveis de decisão, uma vez que $Y_{i}(\bar{\alpha}(k)), i=1,2,3$, são matrizes dadas a priori. No entanto, como discutido em Felipe et al. (2016), fixar os valores de $Y_{i}(\bar{\alpha}(k))$ reduz as chances do Teorema 1 fornecer soluções factíveis. Para utilizar o resultado de maneira mais eficaz, propõe-se um algoritmo iterativo para resolver o teorema e sugere-se a inclusão de uma relaxação na condição de estabilização, a qual pode ser vista como uma extensão para o problema de controle de sistemas LPV discretos da abordagem apresentada em Felipe et al. (2016) (que trata apenas da estabilização de sistemas LTI politópicos contínuos no tempo). Essa relaxação consiste em resolver (5) substituindo-se $A_{c l}(\alpha(k))$, por $\bar{A}(\alpha(k))=(1-r) A_{c l}(\alpha(k))$, com $r \in(-\infty, 1]$. Note que, $\bar{A}(\alpha(k))=A(\alpha(k))(1-r)+B(\alpha(k)) L(\alpha(k))(1-$ $r) C(\alpha(k))=A(\alpha(k))(1-r)+B(\alpha(k)) \hat{L}(\alpha(k)) C(\alpha(k))$, ou seja, com uma simples mudança de variável, $r$ e $\hat{L}(\alpha(k))$ aparecem de forma linear. Observe que a estabilidade de $\bar{A}(\alpha(k))$ assegura que $1 /(1-r)$ é um limitante superior para o máximo valor absoluto dos autovalores de $A_{c l}(\alpha)$ no caso LTI e para a taxa de decaimento no caso LPV. Isso significa que, para $r \in(0,1]$, garantir a estabilidade de $\bar{A}(\alpha(k))$ é menos restritivo que garantir a estabilidade de $A_{c l}(\alpha(k))$. Por outro lado, se $r \leq 0$, a estabilidade de $\bar{A}(\alpha(k))$ implica a estabilidade de $\overline{A_{c l}}(\alpha(k))$.

Em seguida, é demonstrado que ao empregar essa relaxação e a escolha particular de $\mathcal{B}(\bar{\alpha}(k))=\mathcal{B}_{0}=$ $\left[\begin{array}{lll}0 & I & -I\end{array}\right]$, o Teorema 1 sempre produz soluções factíveis com $P(\alpha(k)) \succ 0$. Assim, escolhendo $r=1(\bar{A}(\alpha(k))=0)$, $\mathcal{X}(\bar{\alpha}(k))=-1 / 2 \mathcal{B}_{0}^{\prime}$, obtém-se

$$
\left[\begin{array}{ccc}
-P(\alpha(k+1)) & 0 & 0 \\
0 & P(\alpha(k))-I & 0 \\
0 & 0 & -I
\end{array}\right] \prec 0 .
$$


Como sempre existe $P(\alpha(k))=P(\alpha(k+1))=\rho I, \rho \in(0,1)$ tal que (9) seja satisfeita, é possível afirmar que $\mathcal{B}_{0}=$ $\left[\begin{array}{lll}0 & I & -I\end{array}\right]$ é uma escolha inicial que garante a factibilidade de (5). Adicionalmente, se $A(\alpha(k)) \geq 0$ (sistema positivo em malha aberta), ao menos $L(\alpha(k))=0$ garante que $(6)$ seja válida.

O procedimento iterativo para o cômputo do ganho $L(\alpha(k))$ descrito no Algoritmo 1 consiste em minimizar $r$ sujeito a (6) e à condição de estabilidade (5) substituindose $A_{c l}(\alpha)$ por $\bar{A}(\alpha(k))$ usando o valor de $\mathcal{B}(\bar{\alpha}(k))=\mathcal{B}_{0}$ sugerido anteriormente. Se $r>0$, o controlador encontrado não é estabilizante e, então, atualiza-se a matriz $\mathcal{B}(\bar{\alpha}(k))$ com o valor computado para $\mathcal{X}^{\prime}(\bar{\alpha}(k))$. Essa atualização pode ser realizada pois $\operatorname{He}(X(\bar{\alpha}(k)) B(\bar{\alpha}(k)))=$ $\operatorname{He}\left(\mathcal{B}^{\prime}(\bar{\alpha}(k)) \mathcal{X}^{\prime}(\bar{\alpha}(k))\right)$. Assim, todos possíveis valores de $\mathcal{X}^{\prime}(\bar{\alpha}(k))$ são escolhas válidas para $\mathcal{B}(\bar{\alpha}(k))$, garantindo que existe solução para as condições do Teorema 1 e que o valor de $r$ nunca cresce ao longo das iterações (no máximo mantém-se igual). Se $r \leq 0$, o ganho $L(\alpha(k))$ é estabilizante e garante a positividade do sistema, finalizando o procedimento iterativo. O Algoritmo 1 também utiliza como critério de parada um número máximo de iterações $i t_{\max }$ definido a priori.

$\overline{\text { Algoritmo } 1 \text { Procedimento iterativo para estabilização }}$ de sistemas LPV positivos discretos no tempo

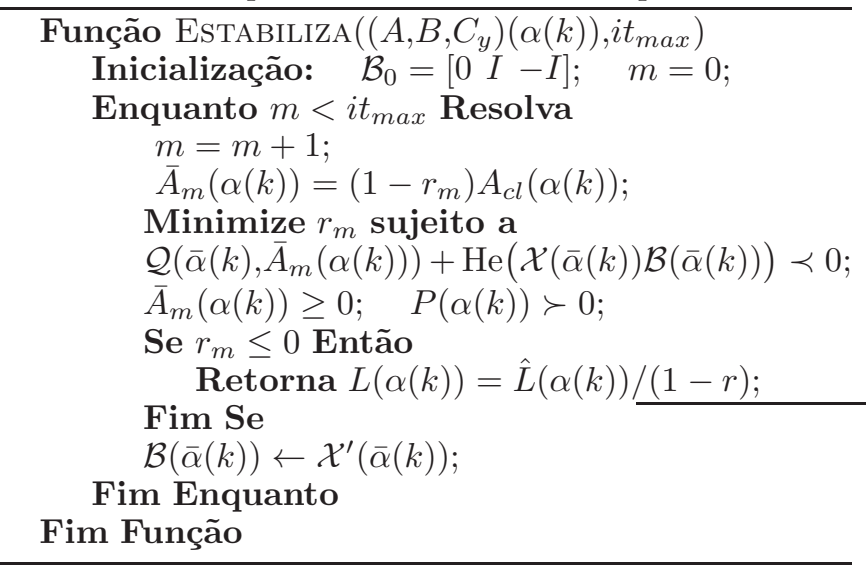

\section{OBTENÇÃO DAS LMIS PROGRAMÁVEIS}

Neste artigo, os parâmetros $\alpha(k)$ são considerados arbitrariamente rápidos, isto é, $\alpha(k+1)$ não depende de $\alpha(k)$ e portanto pode ser considerado como $\alpha(k+1)=\beta(k) \in \Lambda$. Além disso, como as condições (5) e (6) devem ser resolvidas para todo $\alpha(k) \in \Lambda, k \geq 0$, o Teorema 1 constitui um problema de dimensão infinita (teria que ser testado em um número infinito de pontos do domínio). Para resolver as condições do teorema, são utilizadas aproximações polinomiais (relaxações LMIs), ou seja, é testado um conjunto finito de condições LMIs suficientes descritas em termos de polinômios homogêneos (Oliveira and Peres, 2007). A tarefa de obter LMIs suficientes pode ser realizada automaticamente por meio do parser ROLMIP (Robust LMI Parser) (Agulhari et al., 2019). As variáveis polinomiais, nos exemplos apresentados na próxima seção, $P(\alpha(k))$, $P(\alpha(k+1))$ e $\mathcal{X}(\bar{\alpha}(k))$ são respectivamente de grau um nos parâmetros $\alpha(k), \beta(k)=\alpha(k+1)$ e $\bar{\alpha}(k)=(\alpha(k), \beta(k))$. Para o projeto de ganhos escalonados utiliza-se grau um na variável $L(\alpha(k))$, e grau zero na síntese de ganhos robustos (independentes de parâmetros). Todas as rotinas foram programadas em Matlab (R2017a) empregando os parsers ROLMIP (Agulhari et al., 2019) e Yalmip (Löfberg, 2004) e o resolvedor Mosek (Andersen and Andersen, 2000).

\section{EXEMPLO}

Considere o projeto de um ganho robusto por realimentação de estados para estabilizar o sistema (1) cujos vértices das matrizes são

$$
A_{1}=\left[\begin{array}{ll}
6,29 & 3,58 \\
5,28 & 2,79
\end{array}\right], A_{2}=\left[\begin{array}{cc}
1,11 & a \\
2,37 & 2,35
\end{array}\right], B_{1}=\left[\begin{array}{l}
7 \\
6
\end{array}\right], B_{2}=\left[\begin{array}{l}
b \\
3
\end{array}\right] .
$$

O objetivo deste exemplo é comparar o conservadorismo do Algoritmo 1 (A1), usando diferentes estruturas $(s=$ simétrica, $d=$ diagonal e $c=$ arbitrária) nas variáveis de folga $X_{i}(\bar{\alpha}(k)), i=1,2,3$ do Teorema 1 , com as condições (24), (25) e (27) de Ebihara et al. (2014) (respectivamente chamadas de EPA24, EPA25 e EPA27) adaptadas para tratar o caso de estabilização de sistemas LPV positivos.

A eficiência dos métodos é avaliada pela família de sistemas variantes no tempo que podem ser estabilizados, ou seja, quanto maior a área no plano $a \times b$ relacionada a soluções factíveis, menor é o conservadorismo do método.

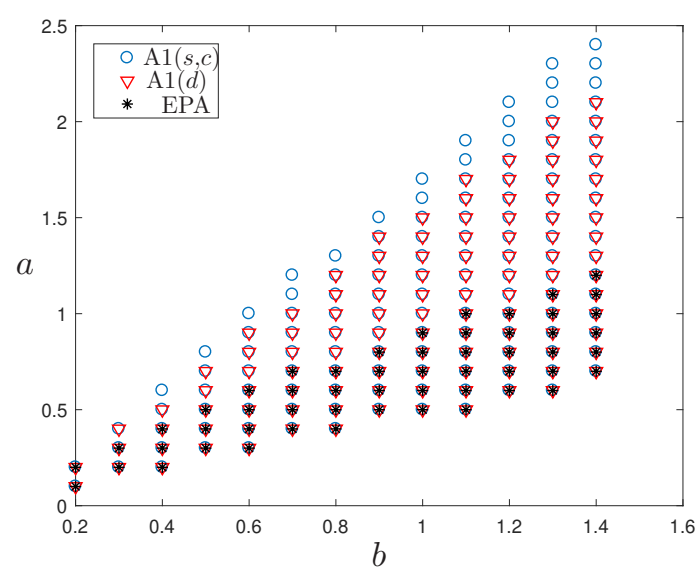

Figura 1. Região de factibilidade obtida com o Algoritmo 1 (A1) utilizando $X_{i}(\bar{\alpha}(k))$ simétrica $(s)$ ou arbitrária (c) (o); diagonal $(d)(\nabla)$; e com Ebihara et al. (2014) - $\operatorname{EPA}(*)$.

É possível observar na Figura 1 que os três métodos de Ebihara et al. (2014) provêm os mesmos resultados, estabilizando um conjunto menor de sistemas que o método proposto neste artigo (Algoritmo 1). Adicionalmente, note que o Algoritmo 1 produz uma maior área de estabilização quando emprega mais variáveis escalares no problema de otimização (estruturas arbitrária e simétrica em vez de diagonal em $\left.X_{i}(\bar{\alpha}(k)), i=1,2,3\right)$.

Quando o valor dos parâmetros variantes no tempo está disponível em tempo real (por medição ou estimação), resultados menos conservadores podem ser obtidos com o emprego de controladores dependentes de parâmetros (escalonados). Diferentemente das condições da literatura, a técnica proposta permite produzir ganhos escalonados com o mesmo algoritmo. A Figura 2 ilustra como a área 
de estabilização obtida pelo Algoritmo 1 (com estrutura simétrica nas variáveis de folga) aumenta significativamente com o emprego de ganhos escalonados.

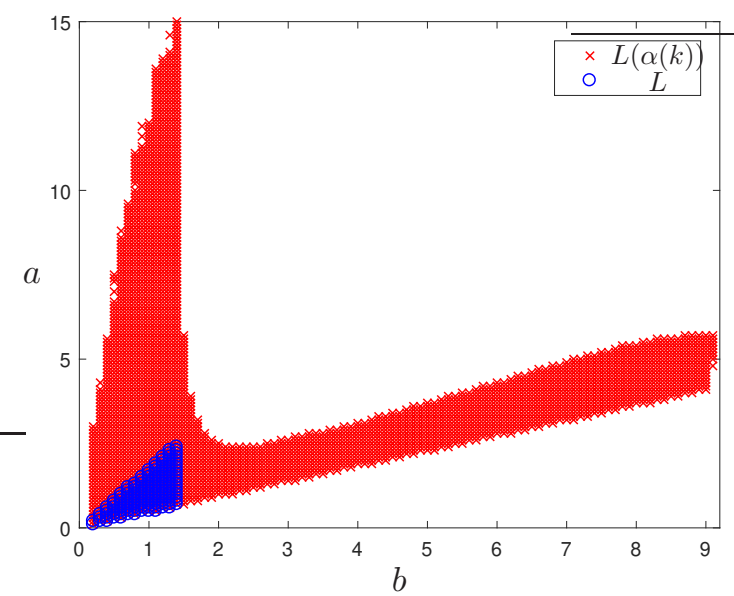

Figura 2. Região de factibilidade obtida com Algoritmo 1 com $X_{i}(\bar{\alpha}(k)), i=1,2,3$ simétricas para a síntese de ganhos escalonados $(L(\alpha(k))-\times)$ e robustos $(L-\circ)$.

Para ilustrar a aplicabilidade do método proposto na síntese de controladores escalonados por realimentação estática de saída, considere $a=2,4, b=1,4 \mathrm{e}$

$$
C_{y_{1}}=\left[\begin{array}{cc}
1 & 0,1 \\
0 & 1
\end{array}\right], C_{y_{2}}=\left[\begin{array}{cc}
1 & 0,4 \\
0 & 1
\end{array}\right] \text {. }
$$

Para esses valores $(a=2,4$ e $b=1,4)$, mesmo considerando o problema de realimentação de estados, os métodos de Ebihara et al. (2014) não são capazes de prover um ganho que estabilize e mantenha a positividade do sistema em malha fechada. Além disso, mesmo que produzissem uma solução factível para o par $(a, b)$ considerando o problema de realimentação de estados, não seria possível adaptar o sistema ou as condições de síntese para computar um controle por realimentação de saída, uma vez que a matriz $C_{y}(\alpha(k))$ é politópica e variante no tempo. Por outro lado, o Algoritmo 1 com $X_{i}(\bar{\alpha}(k)), i=1,2,3$ simétricas, produz o seguinte ganho escalonado ${ }^{1}$

$$
\begin{aligned}
L(\alpha(k))=\alpha_{1}(k)[-0,8800-0,3831] & \\
+ & \alpha_{2}(k)[-0,7900-0,3765],
\end{aligned}
$$

cujos sinais "-" podem ser interpretados como realimentação negativa. O comportamento das trajetórias das saídas e dos estados do sistema (1) em malha fechada com o controlador (11) pode ser visto na Figura 3 para uma condição inicial $x(0)=\left[\begin{array}{ll}1 & 2\end{array}\right]^{\prime}$ e um vetor de parâmetros $\alpha(k)$ que varia de acordo com a seguinte função:

$$
\alpha_{1}(k)=\frac{\operatorname{sen}(0,4 k)+1}{2}, \quad \alpha_{2}(k)=1-\alpha_{1}(k) .
$$

Observe que tanto os estados quanto as saídas em malha fechada são positivos e tendem assintoticamente para zero, enquanto que a saída em malha aberta é instável.

\section{CONCLUSÃO}

O método de estabilização proposto neste artigo difere de outros da literatura pela vantagem de empregar condições

\footnotetext{
1 O projeto robusto não provê solução factível para este caso.
}
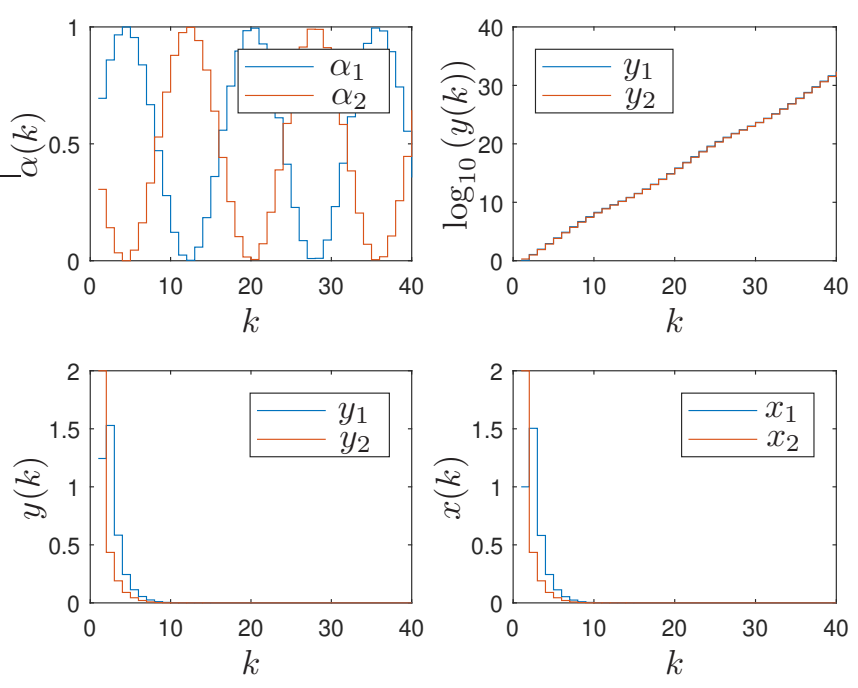

Figura 3. No sentido horário: trajetórias dos parâmetros variantes no tempo $(\alpha(k))$; da saída em malha aberta $\left(\log _{10}(y(k))\right)$; dos estados $(x(k))$ e da saída $(y(k))$ em malha fechada com o ganho (11) para o exemplo.

baseadas em LMIs que tratam os ganhos de controle diretamente como variáveis de otimização. Em contrapartida, é necessário um procedimento iterativo para obter soluções estabilizantes. Apesar do maior custo computacional associado aos repetidos testes de condições LMIs, a adoção de uma dada condição inicial e de uma relaxação na estabilidade garantem soluções factíveis a cada iteração e tornam o método atrativo. No que se refere à estabilização de sistemas LPV positivos discretos, a abordagem proposta mostrou-se eficiente, permitindo estabilizar um conjunto maior de sistemas do que outras técnicas da literatura.

\section{REFERÊNCIAS}

C. M. Agulhari, A. Felipe, R. C. L. F. Oliveira, and P. L. D. Peres. Algorithm 998: The Robust LMI Parser - A toolbox to construct LMI conditions for uncertain systems. ACM Trans. Math. Softw., 45(3):36:1-36:25, August 2019. http://rolmip.github.io.

M. Ait-Rami, B. Boulkroune, A. Hajjaji, and O. Pagès. Stabilization of LPV positive systems. In Proc. 53rd IEEE Conf. Decision Control, pages 4772-4776, Los Angeles, CA, USA, December 2014.

E. D. Andersen and K. D. Andersen. The MOSEK interior point optimizer for linear programming: An implementation of the homogeneous algorithm. In H. Frenk, K. Roos, T. Terlaky, and S. Zhang, editors, High Performance Optimization, volume 33 of Applied Optimization, pages 197-232. Springer US, 2000. http: //www. mosek. com.

A. Benzaouia and F. Tadeo. Output feedback stabilization of positive switching linear discrete-time systems. In Proc. 16th Medit. Conf. Control Aut. (MED2008), pages 119-124, Ajaccio, France, June 2008.

S. Bhattacharyya and S. Patra. Static output-feedback stabilization for MIMO LTI positive systems using LMIbased iterative algorithms. IEEE Control Syst. Letts., 2 (2):242-247, April 2018.

C. Briat. Robust stability and stabilization of uncertain linear positive systems via integral linear constraints: 
$L_{1}$-gain and $L_{\infty}$-gain characterization. Int. J. Robust Nonlinear Control, 23(17):1932-1954, June 2012.

L. Caccetta and V. G. Rumchev. A survey of reachability and controllability for positive linear systems. Ann. Oper. Res., 98(1):101-122, December 2000.

J. Daafouz and J. Bernussou. Parameter dependent Lyapunov functions for discrete time systems with time varying parameter uncertainties. Syst. Control Lett., 43 (5):355-359, August 2001.

W. P. Dayawansa and C. F. Martin. A converse Lyapunov theorem for a class of dynamical systems which undergo switching. IEEE Trans. Autom. Control, 44(4):751-760, April 1999.

J. De Caigny, J. F. Camino, and J. Swevers. Interpolating model identification for SISO linear parameter-varying systems. Mechanical Systems and Signal Processing, 23 (8):2395-2417, November 2009.

J. De Caigny, J. F. Camino, R. C. L. F. Oliveira, P. L. D. Peres, and J. Swevers. Gain-scheduled $\mathcal{H}_{2}$ and $\mathcal{H}_{\infty}$ control of discrete-time polytopic time-varying systems. IET Control Theory \& Appl., 4(3):362-380, March 2010.

G. S. Deaecto and J. C. Geromel. $\mathcal{H}_{2}$ state feedback control design of continuous-time positive linear systems. IEEE Trans. Autom. Control, 62(11):5844-5849, November 2017a.

G. S. Deaecto and J. C. Geromel. $\mathcal{H}_{2}$ state feedback control design of positive switched linear systems. In Proc. 20th IFAC World Congr., pages 3136-3141, Toulouse, France, July 2017b.

Y. Ebihara, D. Peaucelle, and D. Arzelier. $L_{1}$ gain analysis of linear positive systems and its application. In Proc. 50th IEEE Conf. Decision Control - Eur. Control Conf. ECC 2011, pages 4029-4034, Orlando, FL, USA, December 2011.

Y. Ebihara, D. Peaucelle, and D. Arzelier. Optimal $L_{1}$ controller synthesis for positive systems and its robustness properties. In Proc. 2012 Amer. Control Conf., pages 5992-5997, Montreal, QC, Canada, June 2012.

Y. Ebihara, D. Peaucelle, and D. Arzelier. LMI approach to linear positive system analysis and synthesis. Syst. Control Lett., 63:50-56, January 2014.

Y. Ebihara, P. Colaneri, and J. C. Geromel. $H_{2}$ statefeedback synthesis for discrete-time systems under positivity constraint. In Proc. SICE 2018, pages 39-46, Tokyo, Japan, March 2018.

L. Farina and S. Rinaldi. Positive Linear Systems: Theory and Applications. John Wiley \& Sons, New York, NY, 2000.

A. Felipe, R. C. L. F. Oliveira, and P. L. D. Peres. An iterative LMI based procedure for robust stabilization of continuous-time polytopic systems. In Proc. 2016 Amer. Control Conf., pages 3826-3831, Boston, MA, USA, July 2016.

L. Gurvits, R. Shorten, and O. Mason. On the stability of switched positive linear systems. IEEE Trans. Autom. Control, 52(6):1099-1103, June 2007.

E. Hernandez-Vargas, P. Colaneri, R. Middleton, and F. Blanchini. Discrete-time control for switched positive systems with application to mitigating viral escape. Int. J. Robust Nonlinear Control, 21(10):1093-1111, May
2011.

C. Hoffmann and H. Werner. A survey of linear parametervarying control applications validated by experiments or high-fidelity simulations. IEEE Trans. Control Syst. Technol., 23(2):416-433, March 2015.

P. N. Kvieska, M. Aït-Ahmed, and G. Lebret. LPV systems: Theoretical results for gain scheduling. In Proc. 2009 Eur. Control Conf., pages 3166-3171, Budapest, Hungary, August 2009.

J.-W. Lee. On uniform stabilization of discrete-time linear parameter-varying control systems. IEEE Trans. Autom. Control, 51(10):1714-1721, October 2006.

J.-W. Lee and G. E. Dullerud. Uniform stabilization of discrete-time switched and Markovian jump linear systems. Automatica, 42(2):205-218, February 2006.

V. J. S. Leite and P. L. D. Peres. Robust control through piecewise Lyapunov functions for discrete timevarying uncertain systems. Int. J. Control, 77(3):230238, February 2004.

J. Löfberg. YALMIP: A toolbox for modeling and optimization in MATLAB. In Proc. 2004 IEEE Int. Symp. on Comput. Aided Control Syst. Des., pages 284-289, Taipei, Taiwan, September 2004. http://yalmip.github. io.

D. G. Luenberger. Introduction to Dynamic Systems. Theory, Models \& Apllications. John Wiley \& Sons, Inc., New York, NY, 1979.

O. Mason and R. Shorten. On linear copositive Lyapunov functions and the stability of switched positive linear systems. IEEE Trans. Autom. Control, 52(7):13461349, July 2007.

V. F. Montagner, R. C. L. F. Oliveira, V. J. S. Leite, and P. L. D. Peres. Gain scheduled state feedback control of discrete systems with time-varying uncertainties: An LMI approach. In Proc. 44th IEEE Conf. Decision Control - Eur. Control Conf. ECC 2005, pages 43054310, Seville, Spain, December 2005.

F. Najson. On the Kalman-Yakubovich-Popov lemma for discrete-time positive linear systems: A novel simple proof and some related results. Int. J. Control, 86(10): 1813-1823, 2013.

R. C. L. F. Oliveira and P. L. D. Peres. Parameterdependent LMIs in robust analysis: Characterization of homogeneous polynomially parameter-dependent solutions via LMI relaxations. IEEE Trans. Autom. Control, 52(7):1334-1340, July 2007.

A. Rantzer. Distributed control of positive systems. In Proc. 50th IEEE Conf. Decision Control - Eur. Control Conf. ECC 2011, pages 6608-6611, Orlando, FL, USA, December 2011.

J. Shen and J. Lam. Some extensions on the bounded real lemma for positive systems. IEEE Trans. Autom. Control, 62(6):3034-3038, June 2017.

R. Shorten, O. Mason, and C. King. An alternative proof of the Barker, Berman, Plemmons (BBP) result on diagonal stability and extensions. Lin. Alg. Appl., 430(1):34-40, January 2009.

T. Tanaka and C. Langbort. The bounded real lemma for internally positive systems and H-infinity structured static state feedback. IEEE Trans. Autom. Control, 56 (9):2218-2223, September 2011. 